\title{
A Dual Switched-Capacitor Integrator Architecture For Versatile, Real-Time Amperometric Biosensing
}

\author{
Michail Pligouroudis, Konstantinos I. Papadimitriou, Daniel Evans and Themistoklis Prodromakis \\ Nanoelectronics \& Nanotechnology Research Group, School of Electronics and Computer Science \\ University of Southampton, Southampton, SO17 1BJ, UK, Email: mp3n14@ecs.soton.ac.uk
}

\begin{abstract}
In this paper, a versatile, re-programmable, current-input bioinstrumentation board is presented for electrochemical amperometric measurements. The proposed instrument has been fabricated on a six layer printed circuit board (PCB) and exploits dual switched-capacitor (SC) integration and sampleand-hold (SH) techniques. It comprises off-the-shelf switch and amplifier ICs and a commercially available FPGA-based DSP unit for digital signal control and synchronisation. It features eight amperometric channels, has a dynamic current range of $100 \mathrm{~dB}$, can be powered-up by a USB port or a $5 \mathrm{~V}$ battery and is portable, with dimensions of $110 \times 110 \mathrm{~mm}^{2}$. An onboard digital-to-analog converter (DAC) combined with standard three-electrode potentiostats can provide precise, programmable biasing voltages to eight amperometric biosensors simultaneously. Validation of the robustness and accuracy of the proposed system is demonstrated by proof-of-concept amperometric measurements using a high-precision Keithley 6221 current source and $\mathrm{NaCl}$ solution on a PCB-based sensor.
\end{abstract}

Keywords-Dual-Switched Capacitor Integration, FPGA, PCBBased Sensor, Point-of-Care Diagnostics, Switched-Capacitor

\section{INTRODUCTION}

The need for diagnostic systems that can identify biomarkers at very low concentrations, e.g. $\mathrm{pg} / \mathrm{mL}$, is constantly rising, as prognostic and diagnostic biodetection systems are becoming more sensitive and more specific for various types of diseases. Driven by modern advances in biochemistry, the concept of a point-of-care (PoC) laboratory remains an interesting and challenging aspect of clinical diagnosis. The benefits of PoC laboratories are multidimensional, especially now that the sustained expansion of the Internet of Things allows for rapid diagnosis and efficient transmission of biological data from anywhere [1]. A significant number of approaches to a large variety of disease diagnostics have been attempted at $\mathrm{PoC}$, exploiting different techniques and materials. The lab-on-aPCB (LoPCB) approach is an alternative electrochemical detection method, using amperometric sensors and microfluidics produced by standard PCB manufacturing processes [2]-[4].

Inspired by the LoPCB framework for electrochemical detection, the concept of a handheld, electronic EnzymeLinked Immuno-Sorbent Assay ("eELISA") has been demonstrated as the next step in future diagnostic technology [5]. The "eELISA" uses passive PCB-based sensors, where the molecular reaction takes place, combined with high-precision, multi-channel, portable bioinstrumentation platforms. In the previous "eELISA" work, the bioinstrumentation platform has been designed based on a low-noise transimpedance amplifier (TIA) front-end combined with high-resolution voltage-input analog-to-digital converters (ADCs). However, it should be stressed that for small current levels (between few tenths of pA up to few nA), traditional TIA setups require a high-value feedback resistor (typically hundreds of $\mathrm{M} \Omega \mathrm{s}$ up to several $\mathrm{G} \Omega \mathrm{s}$ ) for adequate signal detection. Such feedback resistor values usually compromise the signal's integrity due to the effect of thermal noise that they generate. Moreover, the high cost and large area of high value resistors are not always compatible with portable, low-cost $\mathrm{PoC}$ diagnostic platforms.

Herein, we introduce a novel paradigm for the "eELISA" detection method, exploiting the idea of dual SC integration for continuous current measurement [6], [7]. With the use of lowcost, small-size SMD capacitors combined with inexpensive switch ICs, fully reconfigurable SC-based topologies have been designed, providing high dynamic current detection range. Taking into consideration the need for continuous biosignal measurement, this endeavour exploits the dual SC integration technique, ensuring continuous current flow from the PCBbased sensor, without interrupting its biasing. The instrument features eight amperometric channels, each comprised of two identical branches and four distinct stages: (a) dual SC integration, (b) $\mathrm{SH}$, (c) amplification and finally (d) filtering. Section II demonstrates the proposed architecture of the bioinstrumentation platform and Section III presents experimental data conducted with a high-precision Keithley 6221 current source and $\mathrm{NaCl}$ solution on a PCB-based sensor. Section IV discusses future prospects for the presented detection method.

\section{BOARD'S ARCHITECTURE AND IMPLEMENTATION}

\section{A. Circuit Analysis}

For ultra-low noise, high-precision current measurements, the SC paradigm is preferable to a conventional TIA topology, mainly due to the absence of the ohmic resistor and the significant levels of thermal noise that TIAs introduce once highvalue transimpedance resistors are employed. In the SC case, current is moving by charging and discharging appropriate value capacitors, sunchronised by appropriate clock pulses that open or close various switches of the SC topology [8], [9].

In Figure 1, a simplified schematic diagram of a single channel based on the proposed architecture for continuous amperometric measurements is presented. There are four distinct stages in each channel. The first stage includes the dual SC integrators (integrators $\mathrm{A}$ and $\mathrm{B}$ ). Two identical branches, controlled by non-overlapping clocks $F_{1}$ and $F_{2}$ ensure constant current flow, as in every clock cycle the current is switched from one branch to the other. As illustrated in Figure 1, integrators A and B are operating in a complementary manner. Focusing only on the upper integrator, integrator A, its two states of operation are depicted in Figure 1, inset (I). 


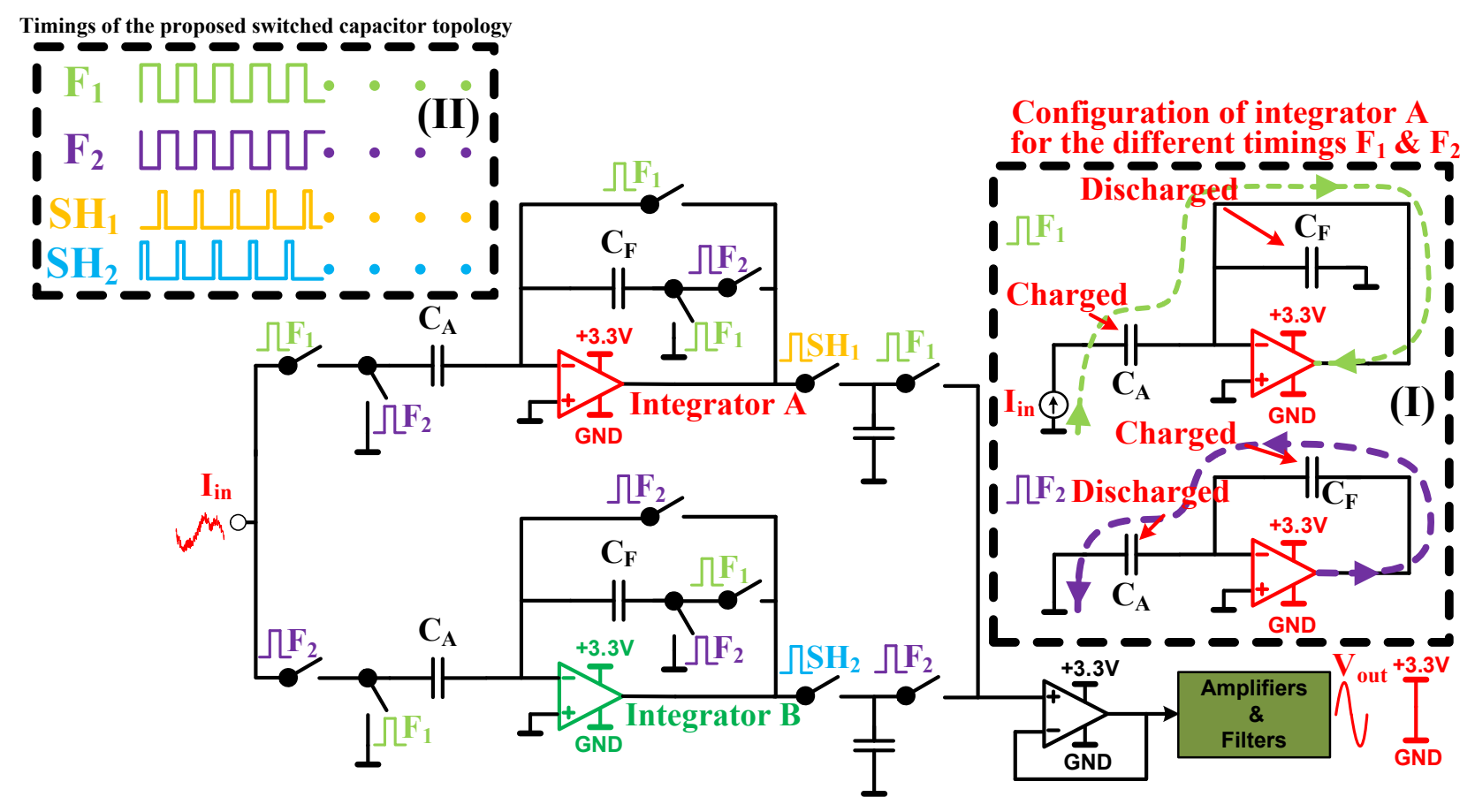

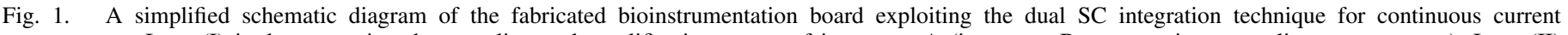

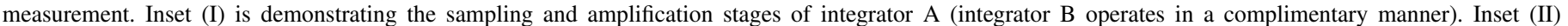
shows the timing between the FPGA control signals.

A logic high of the $F_{1}$ pulse is closing the upper front switch. At the same time a logic zero is applied to the lower front switch through pulse $F_{2}$, opening the circuit. In that case, current will travel through the upper branch and charge capacitor $C_{A}$. During this phase, the capacitor $C_{A}$ of integrator $\mathrm{B}$ will discharge in a complementary manner. This phase is called the sampling phase of integrator $\mathrm{A}$, since $C_{A}$ is sampling the input current. It is worth mentioning that the right hand side of $C_{A}$ capacitor is always grounded. The voltage that is developed across $C_{A}$ during this phase, because the accumulation of the charge, can be expressed as:

$$
V_{C_{A}}=\frac{1}{C_{A}} \times \int_{t_{0}}^{t_{1}} i(t) \partial t, \quad \text { where } \quad t_{1}-t_{0}=1 / 2 f
$$

where $f$ is sampling frequency. Similarly, when a logic low of $F_{1}$ pulse opens the upper front switch, at the same time a logic high is applied to the lower front switch through pulse $F_{2}$, closing the circuit and steering the current towards integrator $\mathrm{B}$. During this phase, capacitor $C_{A}$ of integrator $\mathrm{A}$ is discharging and capacitor $C_{F}$ is charging. The charge that was stored in capacitor $C_{A}$ is now "transferred" to capacitor $C_{F}$. The capacitance of $C_{F}$ has been selected to be lower compared to $C_{A}$. Since it is charged with the same charge as $\mathrm{C} 1$, higher amplified voltage will appear on its terminals. For this reason, this phase is called amplification phase. By changing the capacitance ratio, different amplifications could be achieved and the output voltage can be calculated using equation (2). At this phase, the capacitor $C_{A}$ of integrator $\mathrm{B}$ is charging and $C_{F}$ is discharging in a complimentary manner.

$$
V_{\text {out }}=\left(C_{A} / C_{F}\right) \times V_{C_{A}}
$$

The circuit topology of both integrators is called non-inverting SC amplifier, since $V_{\text {out }}$ has the same polarity as $V_{\text {in }}$ and the gain is greater than unity, if $C_{A}>C_{F}$ [8], [9]. For each channel, three different capacitor values for $C_{A}$ and $C_{F}$ have been placed, resulting into three fixed gain ranges $(\times 2)$. As will be shown later, this setup provides a wide dynamic current range of detection, starting from a few $\mathrm{nA}$ to hundreds of $\mu \mathrm{A}$. At the end of integrators $\mathrm{A}$ and $\mathrm{B}$, a dual $\mathrm{SH}$ circuit has been placed (stage 2), whose switches are controlled by the $S H_{1}$ and $S H_{2}$ pulses of the FPGA. The SH capacitors store the output charge stemming from the two independent integrators, applying a constant SC output voltage at the input of a unity-gain amplifier. Stages 3 and 4 consist of reconfigurable amplification and filtering circuits, respectively. Stage 3 provides three amplification factors $(\times 1, \times 2, \times 5)$ for non-inverting amplification. Finally, stage 4 consists of a $2^{\text {nd }}$-order Sallen-Key low pass filter, whose cut-off frequency ranges from 1 to $500 \mathrm{~Hz}$, removing unwanted switching noise.

\section{B. Fabricated Platform}

A 6-layer PCB has been fabricated for testing the circuit architecture shown in Section II-A. The board features 8 dual integration SC amplifier channels and a 5V single-supply operation. A photo of the fabricated system can be found in Figure 2. The low-voltage quad switches ADG812 from Analog Devices have been selected, mainly due to their small area $\left(5 \times 4.5 \mathrm{~mm}^{2}\right)$, low $R_{O N}$ resistance $(0.5 \Omega)$ and low leakage currents (typically around few hundred pA). The LTC2055 zero-drift amplifier from Linear Technology has been selected for integrators A and B of each channel. The input bias current of this IC is typically around $\pm 3 \mathrm{pA}$.

The period of switching signals $F_{1}$ and $F_{2}$ has been set at least $\times 10$ smaller than the finite gain bandwidth of the amplifier and sufficient settling time has been given. In 


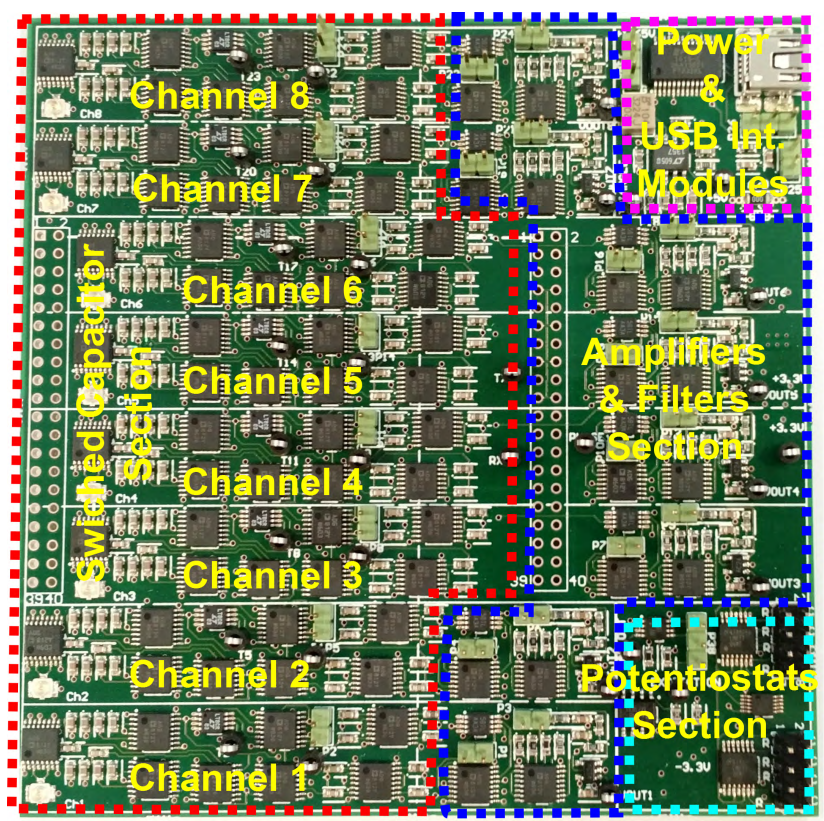

Fig. 2. The dual SC integrators-based bioinstrumentation platform, with its four distinct compartments highlighted.

addition, the slew rate of the amplifier guaranteed its ability to track the switches sufficiently. Finally, with the use of the 12bit, single-output DAC, AD5321, and an appropriate circuitry, the bioinstrumentation board provides bipolar biasing voltages to the sensor from $-3.3 \mathrm{~V}$ up to $+3.3 \mathrm{~V}$ with $\sim 1.6 \mathrm{mV}$ resolution, using a standard three-electrode potentiostat architecture [5]. A summary of the PCB's characteristics is shown in Table I.

TABLE I. BOARD'S CHARACTERISTICS

\begin{tabular}{lc}
\hline Board dimensions $(\mathrm{mm})$ & $110 \times 110$ \\
DSP unit & Altera MAX10 - 50MHz \\
Total current consumption (mA) & $\sim 52$ \\
Power supply & $5 \mathrm{~V}$ (Battery or USB) \\
\hline
\end{tabular}

\section{EXPERIMENTAL RESULTS}

\section{A. Board Characterisation}

For the board's initial characterisation, three separate experiments have been conducted using a fully-controllable, highprecision Keithley $6221 \mathrm{AC} / \mathrm{DC}$ current source. Initially, in order to ensure that the responses of the fabricated circuit comply with the mathematical description of the SC integrator, a comparison between the actual circuit's outputs and a SC simulated model (LTSpice) similar to the one of Figure 1 has been performed for the three ranges provided by the board. DC current inputs were provided to the board with fixed step at each range and the output voltage of each channel was compared to the simulator. The results can be seen in Figure 3. The lines labelled as unoptimised correspond to the circuit's voltage responses when integration time has been set equal to the value used for the simulation. The lines labelled as optimised refer to integration time slightly different than the simulation one, in order to compensate for the circuit's nonidealities (e.g. capacitors have $\pm 20 \%$ tolerances on their nominal values). The optimised circuit responses deviate from ideality no more than $15 \%$, on average, demonstrating the robustness of the system. Finally, the cumulative leakage currents of switch ICs seem to affect only the lower limit of Range 1 and they become negligible as input current increases.

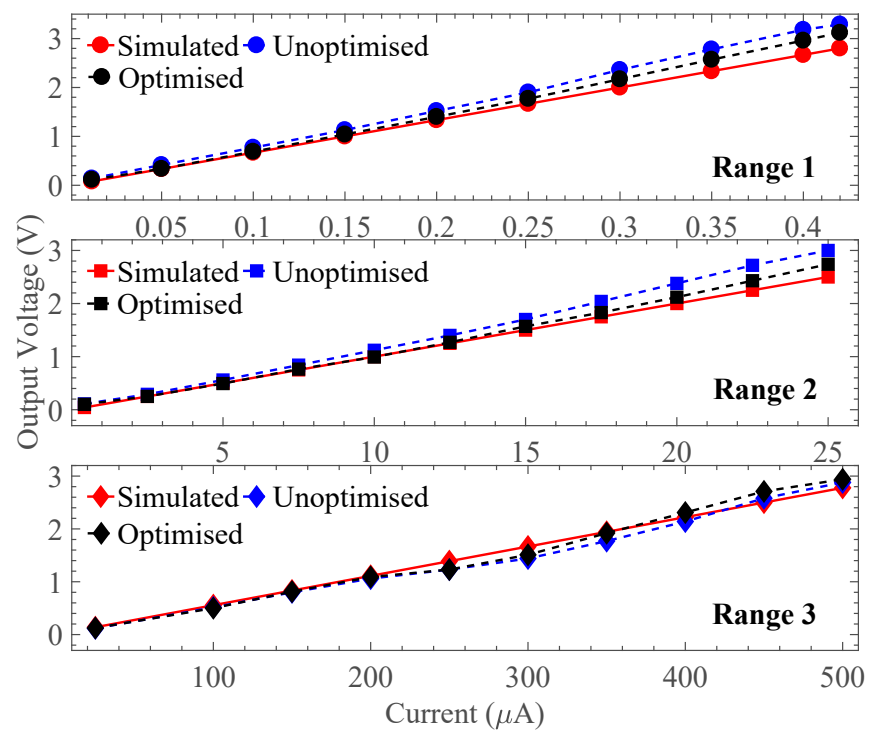

Fig. 3. Comparison between simulations, optimised and unoptimised experimental results of the proposed architecture for three different current ranges.

In the second experiment, the linearity and resolution of the system have been investigated. For this specific experiment, Range 1 (see Table II) has been selected, since this configuration provides the lowest possible current resolution. Current ranges from $20 \mathrm{nA}$ up to $140 \mathrm{nA}$, with step $10 \mathrm{nA}$ and from $30 \mathrm{nA}$ to $60 \mathrm{nA}$ with step $5 \mathrm{nA}$ have been selected. The results are presented in Figure 4. Finally, we demonstrate the noise properties of the system. For each of the three possible range configurations, a sinusoidal current signal has been generated with different amplitude and frequency. The properties of the sinusoidal signals can be found in Table III. The results of the Fast Fourier Transform (FFT) analysis are shown in Figure 5.

TABLE II. SUMMARY OF THE CHARACTERISATION EXPERIMENTS

\begin{tabular}{lccc}
\hline & \multicolumn{3}{c}{ Programmable Current Ranges } \\
\cline { 2 - 4 } & Current Range & Resolution & SC Integration Times \\
Range 1 & $5 \mathrm{nA}-420 \mathrm{nA}$ & $5 \mathrm{nA}$ & {$[\mathbf{U}: 130 \mathrm{~ms}, \mathbf{O}: 125 \mathrm{~ms}]$} \\
Range 2 & $420 \mathrm{nA}-25 \mu \mathrm{A}$ & $100 \mathrm{nA}$ & {$[\mathbf{U}: 2 \mathrm{~ms}, \mathbf{O}: 1.8 \mathrm{~ms}]$} \\
Range 3 & $25 \mu \mathrm{A}-500 \mu \mathrm{A}$ & $1 \mu \mathrm{A}$ & {$[\mathbf{U}: 111 \mu \mathrm{s}, \mathbf{O}: 113 \mu \mathrm{s}]$} \\
\hline Dynamic Current & $20 * \log _{10}\left(\left[500 * 10^{-6} A\right] /\left[5 * 10^{-9} A\right]\right)=100 d B$ \\
Range & \multicolumn{4}{c}{ U:unoptimised, O:optimised }
\end{tabular}

\section{B. Electrochemical Validation}

Electrochemical detection was demonstrated using a titrated series of $\mathrm{NaCl}$ solution samples analysed on a three-electrode PCB-sensor. Electrodes comprise reference $(\mathrm{Ag} \mid \mathrm{AgCl})$, working $(\mathrm{Au})$ and counter $(\mathrm{Au})$ and $\mathrm{NaCl}$ solution samples were measured from $1 \mathrm{M}$ to $125 \mathrm{mM}$ in $\log _{2}$ dilutions. An electrochemical cell was fabricated in PDMS and sealed with PMMA. Fluidic ports through the PMMA top layer allowed sample provision and exchange. Samples were injected rapidly at first $(500 \mu \mathrm{L})$ to ensure complete sample exchange and experimental measurements were taken under flow (syringe pump, $140 \mu \mathrm{L} / \mathrm{min}$ ). The results can be found in Figure 6. 


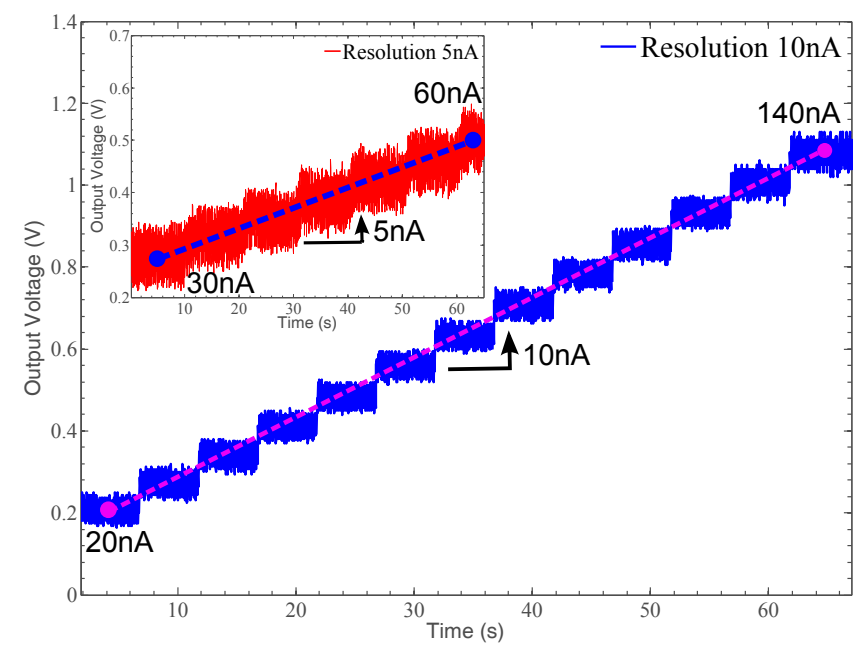

Fig. 4. Linearity and resolution characterisation of the fabricated board.

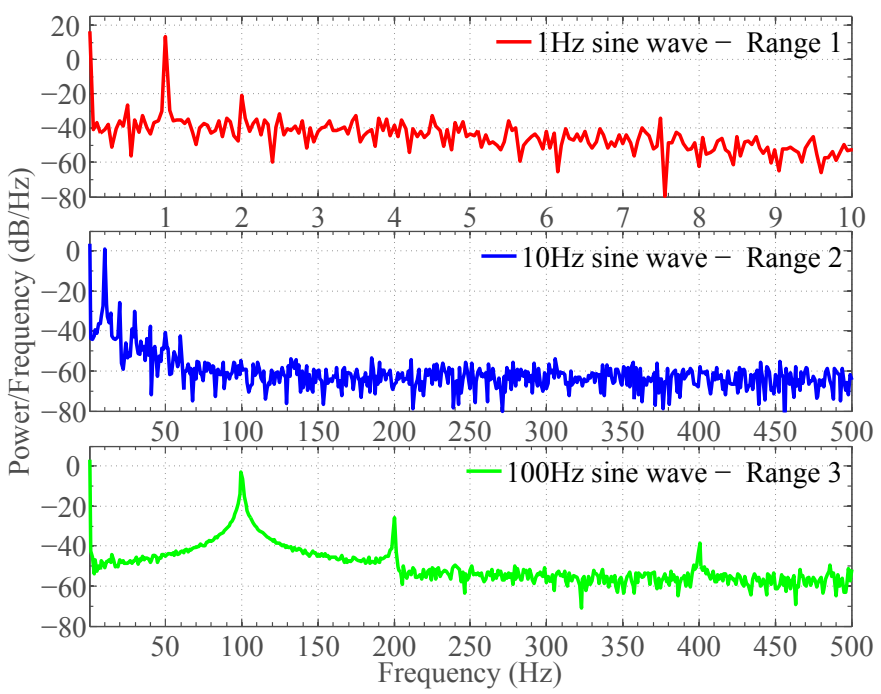

Fig. 5. Noise analysis of each of the three range configurations of the system. The signal's frequency has been selected to be much lower compared to the frequency of the clock signals $F_{1}$ and $F_{2}$.

TABLE III. PROPERTIES OF SINUSOIDAL TEST SIGNAL

\begin{tabular}{lcccc}
\hline & \multicolumn{2}{c}{ Sinusoidal Current Properties } & \\
\cline { 2 - 4 } & $\mathbf{I}_{\mathbf{D C}}$ & $\mathbf{I}_{\mathbf{p}-\mathbf{p}}$ & Frequency & Sampling Frequency \\
Range 1 & $220 \mathrm{nA}$ & $408 \mathrm{nA}$ & $1 \mathrm{~Hz}$ & $1 \mathrm{kHz}$ \\
Range 2 & $13 \mu \mathrm{A}$ & $25 \mu \mathrm{A}$ & $10 \mathrm{~Hz}$ & $1 \mathrm{kHz}$ \\
Range 3 & $260 \mu \mathrm{A}$ & $480 \mu \mathrm{A}$ & $100 \mathrm{~Hz}$ & $1 \mathrm{kHz}$ \\
\hline
\end{tabular}

\section{CONCLUSION}

This paper presents a paradigm shift for the bioinstrumentation platform of the "eELISA" detection approach. The proposed circuitry relies upon the concept of dual SC integration in order to combine continuous current measurement with accuracy and reconfigurability. The fabricated PCB has small footprint and is battery-operated, making it fully-independent from lab instruments or PC control. The obtained experimental results verify our initial hypothesis regarding the lownoise, high-resolution properties of the instrument. Its multiple configuration combinations satisfy the electrochemical requirements of the "eELISA" detection approach. We aspire

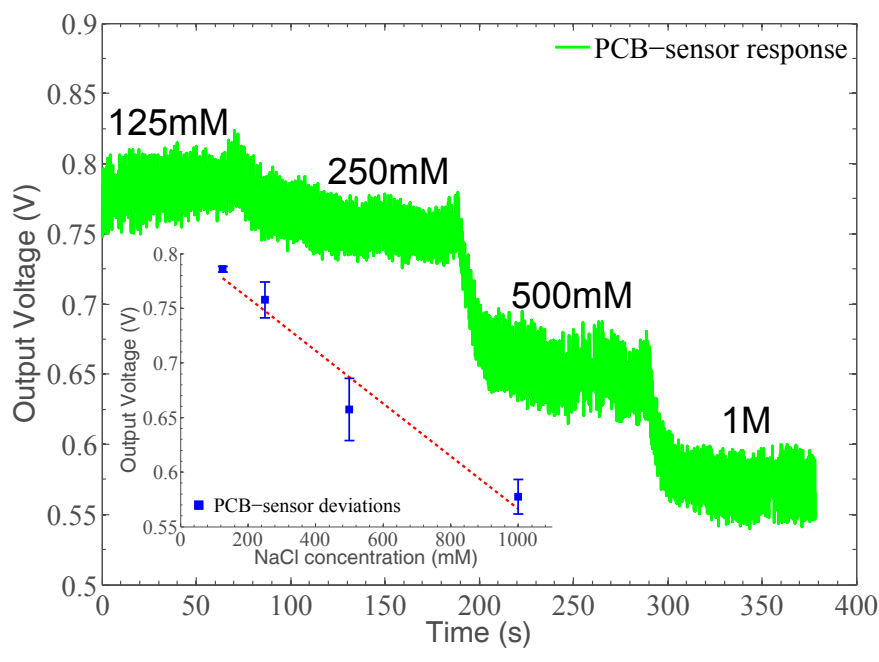

Fig. 6. Electrochemical validation of the proposed board using $\mathrm{NaCl}$ solutions. The system exhibits high degree of linearity with coefficient of determination $\left(R^{2}\right)$ equal to 0.9551 .

to progress our current work by exploiting the Low-Voltage Differential Signalling inputs of FPGA platforms, in order to perform A/D conversion on an FPGA and thus, provide a complete embedded system for PCB-based biosensing.

\section{ACKNOWLEDGMENT}

The authors acknowledge the financial support of the Engineering and Physical Sciences Research Council (EPSRC), EP/L020920/1 research grant.

\section{REFERENCES}

[1] M. Drancourt, A. Michel-Lepage, S. Boyer, and D. Raoult, "The Pointof-Care Laboratory in Clinical Microbiology," Clinical microbiology reviews, vol. 29, no. 3, pp. 429-447, 2016.

[2] N. Vasilakis, K. I. Papadimitriou, D. Evans, H. Morgan, and T. Prodromakis, "The Lab-on-PCB Framework for Affordable, Electronic-Based Point-of-Care Diagnostics: from Design to Manufacturing," in IEEE-NIH 2016 Special Topics Conference on Healthcare Innovations and Pointof-Care Technologies. IEEE, 2016.

[3] T. Prodromakis, Y. Liu, J. Yang, D. Hollinghurst, and C. Toumazou, "A novel design approach for developing chemical sensing platforms using inexpensive technologies," in Biomedical Circuits and Systems Conference (BioCAS), 2011 IEEE, Nov 2011, pp. 369-372.

[4] T. Prodromakis, Y. Liu, and C. Toumazou, "A low-cost disposable chemical sensing platform based on discrete components," Electron Device Letters, IEEE, vol. 32, no. 3, pp. 417-419, 2011.

[5] K. I. Papadimitriou, D. Evans, H. Morgan, and T. Prodromakis, "A PCBbased electronic ELISA system for rapid, portable infectious disease diagnosis," in Biomedical Circuits and Systems Conference (BioCAS), 2016 IEEE.

[6] K. I. Papadimitriou, I. Zeimpekis, D. Moschou, K. Sun, C. Hu, P. Ashburn, H. Morgan, and T. Prodromakis, "Towards a high-precision, embedded system for versatile sensitive biosensing measurements," in Biomedical Circuits and Systems Conference (BioCAS), 2015 IEEE.

[7] K. I. Papadimitriou, C. Wang, M. L. Rogers, S. A. Gowers, C. L. Leong, M. G. Boutelle, and E. M. Drakakis, "High-performance bioinstrumentation for real-time neuroelectrochemical traumatic brain injury monitoring," Frontiers in Human Neuroscience, vol. 10, p. 212, 2016.

[8] M. M. Liu, Demystifying switched capacitor circuits. Newnes, 2006.

[9] B. Razavi, Design of analog CMOS integrated circuits. McGraw-Hill, 2001. 